\title{
Validating Adequacy and Suitability of Business-IT Alignment Criteria in an Inter-Enterprise Maturity Model
}

\author{
Roberto Santana Tapia, Maya Daneva, Pascal van Eck \\ Department of Computer Science \\ University of Twente \\ P.O. Box 217, 7500 AE Enschede, The Netherlands \\ r.santanatapia,m.daneva, p.vaneck@utwente.nl
}

\begin{abstract}
Aligning requirements of a business with its information technology is currently a major issue in enterprise computing. Existing literature indicates important criteria to judge the level of alignment between business and IT within a single enterprise. However, identifying such criteria in an inter-enterprise setting - or re-thinking the existing ones is hardly addressed at all. Business-IT alignment in such settings poses new challenges, as in inter-enterprise collaborations, alignment is driven by economic processes instead of centralized decision-making processes. In our research, we develop a maturity model for business-IT alignment in inter-enterprise settings that takes this difference into account. In this paper, we report on a multi-method approach we devised to confront the validation of the business-IT alignment criteria that we included in the maturity model. As independent feedback is critical for our validation, we used a focus group session and a case study as instruments to take the first step in validating the business-IT alignment criteria. We present how we applied our approach, what we learnt, and what the implications were for our model.
\end{abstract}

\section{Introduction}

Business-IT alignment is the problem of matching services offered by IT with the requirements of the business [27, 38]. In enterprises of any significant size, alignment between business and IT is a hard problem that currently is not completely solved. With the advent of inter-enterprise collaborations, the problem becomes more complex because in such settings, business-IT alignment is driven by economic processes. The wave of inter-

\footnotetext{
* Supported by the Netherlands Organization for Scientific Research (NWO) under contract number 638.003.407 (Value-Based Business-IT Alignment)
}

enterprise collaborations also affects the nature of enterprise computing. Enterprise computing deals with information systems integration across organization boundaries and support inter-organizational processes, communication and collaboration [33]. To achieve efficiency in collaboration, organizations require such distributed information systems $[4,15,26]$ that align with their particular business strategies.

Business-IT alignment can be achieved at various levels of maturity. Therefore, maturity models seem a suitable vehicle for organizations to use in order to gain a deeper understanding of how they progress toward better business-IT alignment. There have been some proposals for alignment maturity models (e.g., [13, 22]). However, as they are oriented to single enterprises, they lack an interenterprise viewpoint that takes specific characteristics of this setting into account. To face this problem, we are developing a Value-based business-IT ALignment Maturity Model, which we call the VITAL MM. The VITAL MM covers all information systems that an organization employs in inter-enterprise collaborations, as well as the technological infrastructure and support facilities needed for them. We refer to these information systems, technological infrastructure, and support facilities, as the organization's information technology (IT).

In an earlier publication [27], we reported on our motivation for developing the VITAL MM, the fundamental principles of its design, the research challenges we met, and the first proposal concerning the business-IT alignment criteria [28] to be included in the model. In this paper, we present the VITAL MM focussing on the need to evaluate this first proposal. Specifically, our goal is to investigate two aspects of the VITAL MM, namely the suitability and the adequacy of the criteria included in it.

The paper describes how we used a focus group session and a case study to validate the model. This validation represents the first step out of the many steps we planned to 
find the criteria that should ultimately be included in our maturity model. Because the details of the model have not been elaborated yet, it is not feasible to prove the validity of the model at this time. Instead, we do an early validation in order to gain initial understanding of how complete and suitable the current business-IT alignment criteria are.

In the rest of this section, we first elaborate on the nature of inter-enterprise collaborations, then we present the business-IT alignment concept in such collaborations, and finally we summarize maturity models literature. This serves as background for the rest of the paper, which is organized as follows: in Section 2, we briefly present (i) the validation aspects of a maturity model, (ii) the first proposal of the business-IT alignment criteria included in the model, and (iii) the validation instruments we selected. Section 3 describes our validation process. Section 4 and Section 5 discuss how we used the focus group session and the case study to evaluate the VITAL MM's criteria. Section 6 discusses the results of the validation approach. Finally, Section 7 concludes the paper.

\subsection{Inter-enterprise collaborations}

Changes in the business environment force enterprises to re-think the way they are doing business. More and more organizations nowadays take advantage of the next level of reengineering approaches which capitalize on connecting and aligning one enterprise's business and IT operations with other enterprises to meet organizational goals. We define an inter-enterprise collaboration to be any "mix-and-match" web of profit-and-loss responsible business units, or of independent companies, connected by IT that work together for a common purpose for a specific period of time.

As inter-enterprise collaborations are enabled by IT, distributed enterprise applications are necessary. Examples of such applications are the multi-stakeholder distributed systems (MSDS) [8]. These are distributed enterprise systems in which components are designed, owned, or operated by distinct loosely coupled stakeholders with possibly conflicting interests [10]. An example of a MSDS is an online travel agency where, although some of the online services are developed by the travel agency itself, it requires services owned by third parties, e.g., payment processing services, flight bookings, and car rentals.

\subsection{Business-IT alignment}

As previously stated, matching services offered by IT with the requirements of the businesses in inter-enterprise settings is a significant problem because there is no single decision point. That is, different decisions are often taken at different times, by different stakeholders, with different decision criteria in mind; and these have to be coordinated.

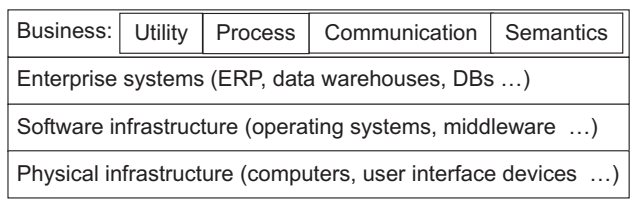

Figure 1. Business-IT alignment framework.

We analyze the business-IT alignment concept in interenterprise collaborations based on the scheme shown in Fig. 1. The horizontal layers classify entities in a service provisioning hierarchy in the operational process of a business: physical entities provide services to a software infrastructure, which provides services to enterprise systems, which provide services to businesses. In the business layer, we take four views on businesses: businesses provide services that have a utility, they perform processes, they communicate with one another, and while doing that, they exchange data that has semantics. This framework is derived from different architecture frameworks (e.g., [19, 36, 40]). Our interest is in the upper two layers of the framework, because this is where the business services and systems alignment in inter-enterprise collaborations takes place. These services and their processes do not necessarily have to be carried out by one organization.

\subsection{Alignment maturity models}

Maturity models have been around for almost 15 years. A maturity model is a framework that describes, for a specific area of interest, a number of levels of sophistication at which activities in this area can be carried out. The bestknown maturity model is the software capability maturity model $^{1}$ (SW CMM) proposed by Carnegie Mellon University's Software Engineering Institute. This model identifies, specifically for software production, five levels of software process management sophistication. For each level, the SW CMM describes which processes need to be executed for an organization to be considered working at this level.

Generally, maturity models help organizations to assess a specific area against a norm to identify lacks of efficiency that can have a negative impact on business benefits. In the literature, several architecture alignment maturity models have been proposed, however they do not include the inter-enterprise perspective. For instance, Luftman's strategic alignment assessment [22] presents an approach for determining an organization's business-IT alignment based on six variables, namely skills, technology scope, partnership, governance, competency measurements, as well as communications. Each of these variables is assigned five levels of alignment. Luftman's model also provides a short description of the variables at each level.

\footnotetext{
${ }^{1}$ More information on http://www.sei.cmu.edu/cmm/
} 
The Chief Information Officer (CIO) Council, a consortium of US Federal executive agency CIOs, developed an architecture-specific alignment and assessment guide as well [13]. This guide provides an overview of the integration of enterprise architecture within the information technology investment planning process. It is used to determine to what degree a proposed investment aligns with business strategies.

\section{The VITAL MM and its validation}

\subsection{MM development and validation}

Before using a maturity model for day-to-day operations, it needs to be validated. Questions arise as to how we can judge the fit of the maturity model in real-life interenterprise settings, and how we can determine whether the model we propose is valid and even what validity comprises in this case.

The validation of maturity models received significant attention since the early days of the CMM [25]. Initial validation attempts compared the costs and quality of the software engineering process of organizations who used the model with those who did not. This kind of validation is, however, not always possible, and therefore, validation is still a big challenge for maturity models researchers. With very few exceptions, existing literature offers almost no advice on how to empirically validate a maturity model. Based on recommendations by researchers in empirical software engineering evaluation [21] and requirements engineering [11,37], we must provide evidence that the model is in fact useful, i.e., to investigate it by empirical means in order to understand it, to evaluate it, and to deploy it in proper contexts. This helps to assure the design of a useful model [17].

A valid maturity model is a model that is sufficiently accurate to achieve its purpose [3]. The purpose of our maturity model is to enable assessing business-IT alignment in inter-enterprise collaborations to plan future business-IT alignment actions while evolving toward a culture of process improvement excellence. With this purpose in mind, the VITAL MM will be considered valid if: (i) it allows inter-enterprise collaborations to assess their alignment, and (ii) it provides an in-depth business transition plan for the collaborations including a roll-out of recommendations to make improvements.

Therefore, for us, validity comprises the completeness and suitability of the variables included in our model to reach the goals - (i) and (ii), mentioned in the previous paragraph. The variables are the enablers of business-IT alignment in inter-enterprise settings that will be assessed using the model. The business-IT alignment criteria will be

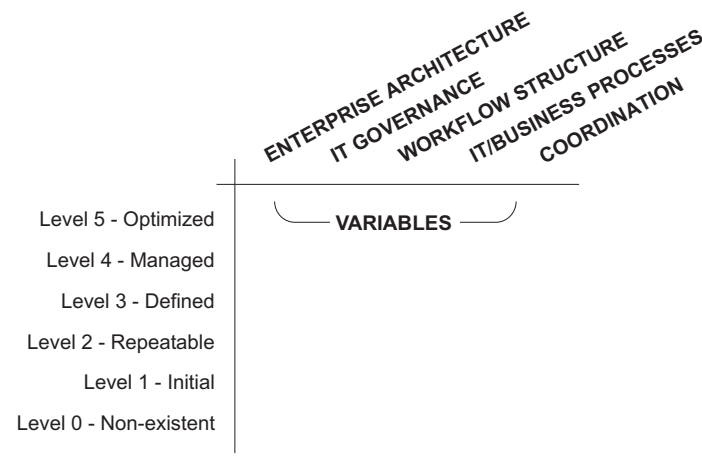

\section{Figure 2. First proposal of the variables of the maturity model.}

complete when it includes all necessary suitable variables that can enable such alignment.

\subsection{The MM to be validated}

This subsection describes the version of our maturity model which we used as input for the validation process. As a result of this process, our maturity model has changed significantly. Section 6 presents our maturity model after changes resulting from the validation efforts had been applied in terms of the new units chosen.

A maturity model has two dimensions (see Fig. 2): the maturity levels and the areas to which these levels are applied $^{2}$. In our context we name those areas 'units'. They are the business-IT alignment criteria, i.e., the main topics to consider to help reaching business-IT alignment. From the literature review (e.g., $[6,13,22]$ ), it is well-known that units such as skills, technology scope, partnership, governance, competency measurements, communications, informal organization, requirements and IT architecture help to align business and IT in single enterprises. Our challenge is to identify such units in inter-enterprise settings.

The version of our maturity model that we used as input for the validation efforts discussed in this paper, as well as the design choices made in its development, has been described extensively elsewhere [28]. Therefore, we only give a short summary of the units of our maturity model, following Fig. 3 from bottom to top:

- Enterprise architecture, defined as the landscape of information systems (IS), the interconnection relations between them, the technology infrastructure on which they run, and the way they create value for the organization.

\footnotetext{
${ }^{2}$ Take for example CMMI [7]. In CMMI the first dimension consists of five levels: initial, managed, defined, quantitatively managed, and optimizing; and the second dimension distinguishes four aspects: process management, project management, engineering, and support.
} 
- IT/business processes, defined as the architecture of all processes needed to reach the shared goals of the collaborating enterprises. These processes are both primary business processes of the collaboration and processes needed for information exchange among the collaborating enterprises.

- Workflow structure, defined as the specification of the roles and responsibilities with respect to the IT and business processes that comprise the previous unit.

- IT governance, defined as the "leadership, organizational structures and processes that ensure that the enterprise's IT sustains and extends the organization's strategies and objectives" [18, p. 5].

- Coordination, defined as the mechanisms to manage the interaction and work among the participating organizations taking into account the dependencies and the shared resources among the IT/business processes.

Fig. 3 illustrates how the enterprise architecture is the "basis" on which both IT and business processes rest. The enterprise architecture is instrumental to perform the collaborative IT/business processes. The definition of these processes in the inter-enterprise collaboration requires that they can interoperate freely [34]. Understanding of the inter-enterprise IT/business processes is an important starting point for the definition of roles and responsibilities [31]. Then, we can say that based on such processes, the workflow structure needs to be defined. This is important because in inter-enterprise settings there is commonly no single central authority. Even with a single authority, in a certain point the inter-enterprise collaboration can become complex and too large for centralized decision making and control [30].

Fig. 3 also shows how workflow structure and IT governance overlap one another. Both units' definitions refer to work division and ownership. However, while IT governance is focused on the IT to support the business strategy, workflow structure is more related to the overall specification of roles and responsibilities. Finally, coordination mechanisms are needed to control all the interactions and the collaborative processes. There are several existing approaches that offer support to our decision on including the five units in our model [28].

We claim that enterprise architecture, IT governance, workflow structure, IT/business processes and coordination are the most important topics to consider when dealing with inter-enterprise collaborations so that value is created for the participating organizations and business-IT alignment is achieved. Using two validation instruments - a focus group session and a case study, we validate the completeness and suitability of the business-IT alignment criteria for interenterprise settings. The next subsection discusses these two validation methods.

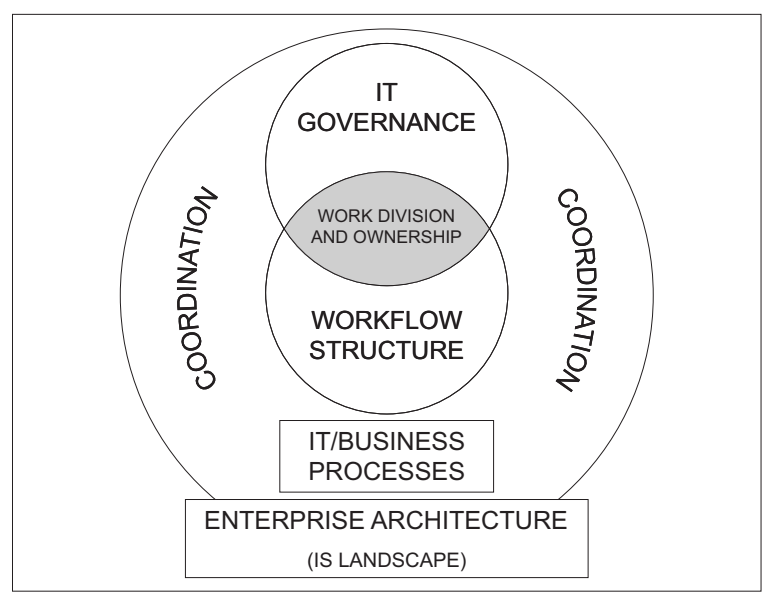

\section{Figure 3. Relationships among units of the model.}

\subsection{Selected validation instruments}

To devise a process for validating the business-IT alignment criteria included in the VITAL MM, we chose two methods preferred by other researchers in their MM validation studies (e.g., [2, 3, 16, 24, 29]). These are a focus group session and a case study. Because each method brings its own unique advantages and limitations, we decided to use them jointly and, thus, ensure the triangulation of our results.

2.3.1. Focus group session. A focus group is a panel of professionals, led by a moderator and guided to exchange ideas, receptions, and experiences on a specific topic [9]. Focus group research is a form of qualitative research. As such, it sacrifices reliability in favor of validity, i.e., focus group results cannot be replicated statistically, they are only useful in extracting data to validate findings. Though, focus group research is a widely popular method, due to its capability to generate a rich understanding of a topic by involving a group of people in an active discussion, rather than by using a single qualitative method, such as a survey [32]. A focus group session is commonly conducted with a group up to 12 professionals; so, it is similar to small samples research.

Focus groups are best-known for their usefulness to validate findings and gather recommendations that can derive changes from existing designs or new hypotheses [9, 32]. We decided to include this method, because of its fit to our research context and our purpose: (a) for $\mathrm{MM}$, people are valuable sources of information and a focus group is proven in provoking attendees to actively articulate their thoughts, perceptions, and behaviors; (b) one of the MM authors can serve as the moderator to help 
attendees retrieve information; (c) the dynamics of a focus group is known to help generate valid and reliable data needed for the validation of artifacts in their early stage of development; and (d) group interviewing is considered to be more effective than individual interviewing in particular research circumstances [16] which we also observed in our context.

2.3.2. Case study research. We decided to couple the use of a focus group session with an exploratory case study research method [21, 39], which is an empirical inquiry aimed at revealing aspects of a contemporary phenomenon inseparable from its real-life context, and thus, difficult to replicate in a laboratory environment. Our motivation for using this method rests on the following: (a) exploratory case studies are particularly appropriate for initial evaluation as they allow the course of the study to be adjusted along the way to account for what is learned; (b) case studies are also the most satisfactory approach when there are many variables of interest and few data points and where resources do not permit enough replications to isolate the variables individually; and (c ) case studies are well-suited to learn about phenomena in real-life context rather than in isolation, and this is exactly the context of the business-IT alignment criteria of interest in our MM [39].

The next section presents how these two methods are complemented in the validation process which we designed and followed.

\section{The validation process}

As shown in Fig. 4, our validation strategy proposes to carry out a focus group session prior to a case study. The rational for this is to get initial qualitative data that would give new insights into the VITAL MM units before beginning to identify these units in the case study. Our approach is consistent with recommendations in leading literature sources $[9,32]$ which point to the use of a focus group session to gain insights into the preliminary results of the design of the model. Then, the researchers use the collected insights to develop new perspectives of the model and derive new hypotheses or changes in the model design. A case study is, then, to be conducted to further clarify the design options worth considering when enhancing the maturity model definition. The main activities involved in the validation process are:

1. Formulate and communicate the purpose of the group session.

2. Bring together professionals.

3. Conduct the focus group presenting the units included in the model.
4. Let professionals speak, share ideas and discuss.

5. Stimulate professionals to move their thoughts from reactions to recommendations.

6. Treat disagreements among professionals by inviting them to re-discuss topics.

7. Register the spoken recommendations.

8. Analyze how recommendations could derive new hypotheses or changes on design.

9. Report results of the focus group session.

10. Formulate and communicate the purpose of the case study.

11. Present the project to our contact partners in the interenterprise collaboration.

12. Receive and classify documentation about the case study from the contact partners.

13. Read the documentation to identify information concerning the model's units.

14. Analyze and report the findings of the case study.

15. Compare case study's findings to the results of the focus group session.

16. Adapt the VITAL MM so that it reflects the results of the validation.

While running the validation process, we kept in mind that the most important task was to constantly watch for the distinction between relevant information and trivial data. We consider relevant those information pieces which tell us something of interest about the connection between the business-IT alignment criteria and the knowledge we possess concerning them. As we wanted to identify in the documentation received in step 12 those information pieces only which related to each of the units included in the VITAL MM, we focused on those documents that presented information related to (i) technology, (ii) processes and (iii) governance. Then, to identify the workflow structure, we referred to the contract and the SLAs. Although no coordination mechanisms were explicitly mentioned in the documents, we could identify them.

The next two sections discuss in detail the two validation instruments we used in our validation strategy. How the insights, which we gained from the focus group session and the case study, impacted our model is discussed in Section 6. 


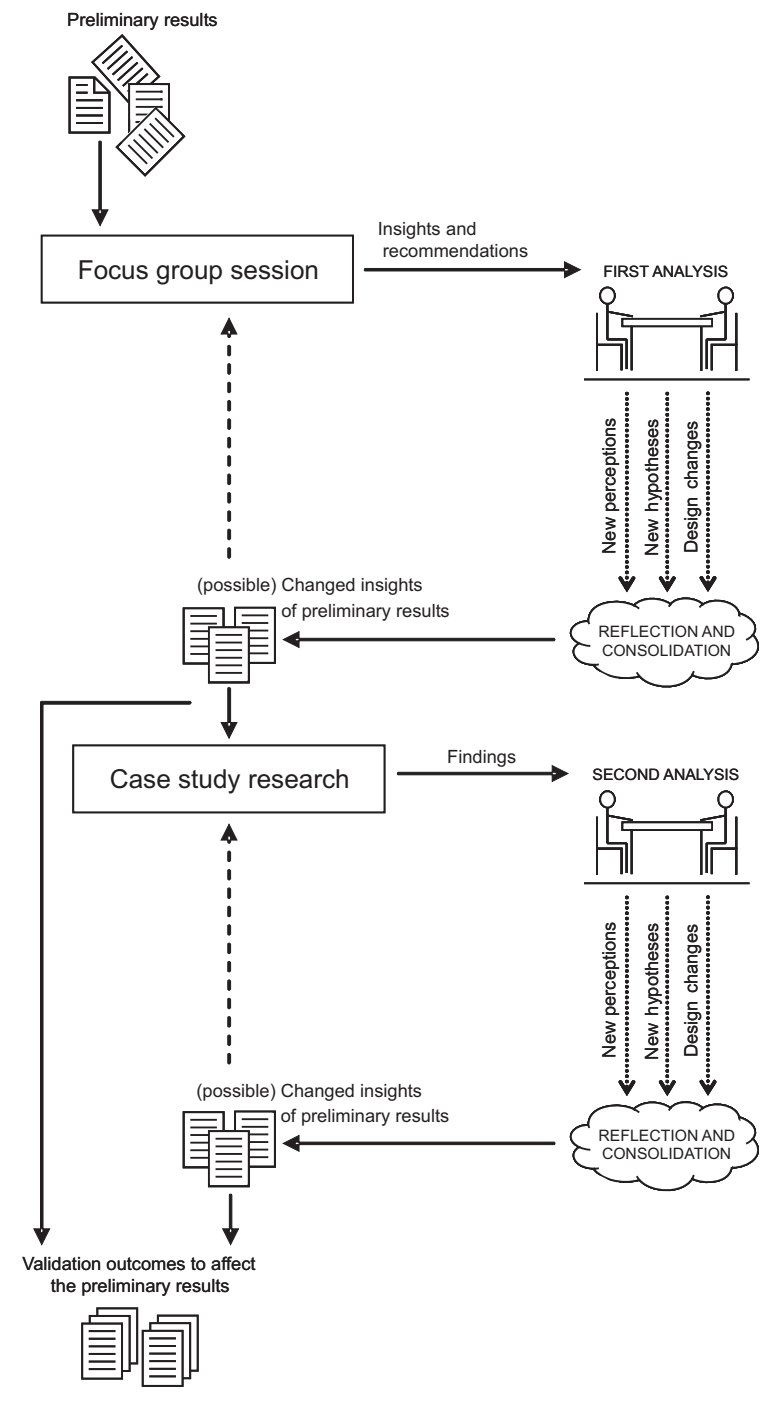

Figure 4. Our maturity model validation approach.

\section{The focus group session}

The goal of our focus group session was to identify initial perceptions of successes, challenges and future research directions for the VITAL MM. Particularly, we wanted to understand how suitable and adequate the units included in the model were. Our focus group session was facilitated by one of us (Santana Tapia) who presented the business-IT alignment criteria we had and the rational for their inclusion in the VITAL MM. Then, we received from the group members their feedback, comments, and suggestions for improvements. The specific results we obtained are presented in Subsection 4.2.

\subsection{Group population}

To set up the focus group work, we emulated a previous study [29] that validated a measurement instrument. We invited professionals to be part of a group to discuss our first results about the design of the VITAL MM. While we targeted professionals from different background as recommended by Kitchenham et al. [20], it turned out that the professionals' availability was the key factor determining their attendance to the focus group session. The professionals were drawn from the Information Systems Group of the University of Twente and the business partners participating in our research project ${ }^{3}$. Table 1 classifies the focus group participants based on their expertise.

\section{Table 1. Distribution of participants' expertise in the focus group session}

\begin{tabular}{l|c|c|c|c|c}
\hline Field of expertise & A & B & C & D & Total \\
\hline \hline Business-IT alignment & 1 & 1 & 1 & & 3 \\
Software systems & & 1 & 2 & 1 & 4 \\
Security & & 1 & 1 & & 2 \\
Workflows & & 1 & 1 & & 2 \\
Cooperative work & & & 1 & & 1 \\
Requirements engineering & & & 2 & & 2 \\
Enterprise architecture & 2 & & & 3 & 5 \\
Coordination processes & 1 & & & & 1 \\
& & & & & \\
Total & 4 & 4 & 8 & 4 & 20 \\
\hline
\end{tabular}

Legend $\mid \begin{aligned} & \mathrm{A}=\mathrm{PhD} \text { candidate } \\ & \mathrm{B}=\text { Postdoc researcher } \\ & \mathrm{C}=\text { Professor } \\ & \mathrm{D}=\text { Business partner }\end{aligned}$

\subsection{Results of the focus group}

Because the VITAL MM is based on the overall structure of Luftman's model, we can say that the participants' opinions on this model and their knowledge of the CMM (the most well-known maturity model proposed by the Software Engineering Institute to assess software capability in organizations) may affect their perception of the VITAL MM. Keeping this in mind, we identified six key points that came out as a result of the focus group session:

1. 9 out of 14 professionals found that in general the unit enterprise architecture covers all the other units.

\footnotetext{
${ }^{3}$ For a complete list of the business partners participating in our research project, please refer to http://www.vital-project.org/
} 
2. Having in mind the definition of enterprise architecture that we presented, professionals thought IS architecture or IS landscape is a better name for this unit.

3. 57 percent of the professionals raised the question: "why to include workflow structure as a separated unit if IT governance includes already some definition of roles and responsibilities?"

4. There was a strong consensus amongst the professionals that the name of the unit workflow structure must change. It is to avoid its confusion with the most common perception of the term workflow, i.e., automation of a business process, in whole or part, during which information is passed from one participant to another based on some rules [1].

5. Professionals indicated that - in their experiences - coordination concepts and IT/business processes could not exist in separation. They found that some coordination mechanisms were always used to glue interenterprise process fragments together and make them run smoothly.

6. A final concern that three professionals had was: why not to consider the collaboration as a single enterprise and then apply Luftman's model for assessing?

The effects that these six concerns have on the VITAL MM are discussed in Section 6. However, in the next section, we first focus on the case study we completed as per the process in Fig. 4.

\section{The case study}

In this section we describe how we set up an exploratory study which examined the collaborative work between two organizations. Its objective was to investigate whether and to what extent the units of the VITAL MM are present in the inter-enterprise collaboration investigated. Specifically, we wanted to identify important information concerning (i) each of the units included in the VITAL MM, and (ii) new important topics to consider in business-IT alignment attempts.

\subsection{Research site}

We selected an inter-enterprise context because our maturity model aims to provide meaningful interpretations of the assessment from the perspective of an entire interenterprise collaboration. That is in contrast with the current alignment maturity models, which focus on a single enterprise. The collaboration we studied is an outsourcing relationship between a leading international business and technology integrator and a local provider of massmarketed services (hereinafter referred to as: Insourcer and Outsourcer, respectively).

Insourcer is an international information technology services company, providing consultancy, systems integration and managed operations. Outsourcer offers a wide range of services for both the private and business market. It focuses on the Benelux-countries and Germany. The stock of both Insourcer and Outsourcer is traded on Euronext, the panEuropean stock exchange, where both companies are in the top 100 in terms of market capitalization.

Outsourcer decided to outsource part of its IT operations to Insourcer in 2001 and this was a measure to confront the company's troubled IS architecture management. The 2001 architecture consisted of home-grown applications, stove pipe solutions, and a lot of point-to-point connections. The company experienced problems related to inconsistent data, significant operational expenses, and below-average customer satisfaction. The outsourcing measure had the objective to help (i) provide continuity of service to the customers so that number of complaints is reduced, (ii) improve financial results due to purchase price and cost reduction, and (iii) optimize the IT architecture and performance.

The relationship between the two organizations is based on a joint-go-to-market approach in the regional market. This means that Insourcer provides consulting, systems integration and managed operations services to Outsourcer, but at the same time it receives services from Outsourcer. It is thought to be a win-win outsourcing relationship. Insourcer is the major supplier for Outsourcer and it delivers almost all of their portfolio assets. The most important assets are datacenter and application management, desktop services, and software house activities. On the other hand, Outsourcer delivers a wide range of services to Insourcer.

\subsection{Data sources}

After our research project was presented to our contacts in Outsourcer, they provided documentation concerning their collaborative work with Insourcer. We found three types of documents useful for the purpose of our case study and we carefully reviewed them. These were:

- Letters and memoranda concerning a variety of business-IT alignment topics in their collaborative work.

- Agendas, minutes and reports of meetings that partners of the two organizations attended to discuss alignment issues and solutions.

- Partner relation management documents, e.g., contracts and SLAs. 
All documents were created in the period 2004-2005, and they were the only ones available to us. According to Yin [39], these types of documents are useful even though they are not always accurate and, though, they can report bias, i.e., they can reflect the bias of the author. Documents must be carefully used and should not be accepted as literally recording of information and events. The documentation we obtained was mostly clear. However, there were some unexplained acronyms and figures. This brought confusion into our interpretations. In total, we received fifteen documents where we could find how the outsourcing partnership arose, what was wrong and what were the agreements on solving the business-IT alignment problems.

\subsection{Findings in the case study}

The documentation was read and analyzed by two of the researchers who are involved in the development of the maturity model. Having done such analysis by ourselves may reflect some bias in how we interpreted the data. We summarize our findings as follows.

5.3.1. Enterprise architecture. The available documents presented just a big picture of the application landscape of Outsourcer to show the organization's IT reality prior to the collaboration with Insourcer. Outsourcer had a homegrown application landscape with stove pipe solutions and a lot of point-to-point connections. The company had inconsistent data, big expenses and could not manage the client service in an appropriated way what caused poor customer satisfaction. Dependencies among the partner's information systems in the entire collaboration were not found.

5.3.2. IT/business processes. The documents showed nothing concerning the processes that each participating organization will perform as collaborative processes. As a result of setting up the outsourcing relation, all tasks that together constitute the joint processes were allocated to the business partners in a clear and consistent way. However, these processes themselves were not documented, at least not in the set of documents available to us. The definition of processes that can be found in the documentation is related to the Outsourcer's processes that were not functioning properly, and it was to have a common understanding on how jointly, Outsourcer and Insourcer could improve such processes.

5.3.3. Workflow structure. The industry sector in which Outsourcer operates is almost constantly changing in a fast pace. The workflow structure of Outsourcer is therefore very complex and constantly changing. Outsourcer addresses this complexity in a 'divide-and-conqueror' way by distinguishing three main IT branches:

1. IT systems that are part of the national service distribution network maintained by Outsourcer,

2. IT products and services offered to their customers, and

3. the IT systems that support the business processes of Outsourcer.

This last IT branch was the first one to be outsourced. After that, Outsourcer's datacenter, end-user support and software house were also outsourced, all to Insourcer. Outsourcer and Insourcer together developed a general governance model and guiding principles for their inter-enterprise collaboration to monitor and control the agreements on rights and responsibilities.

5.3.4. IT governance. What went wrong were the decision making mechanisms (e.g., there was lack of ownership and assigned responsibilities) and the inconsistent level of business commitment (e.g., there was no compliance to agreements and lack of clarity). Therefore, in the process of setting up the collaboration, they defined leadership roles, distributed responsibilities, and identified the activities needed to improve the deployment of the IT potential to support Outsourcer's business objectives, e.g., develop IT management manual and install strategic/tactical IT platforms.

5.3.5. Coordination. In this collaboration, we observed reciprocal dependencies [35] between the two organizations. Such a situation led to establish a considerable number of coordination mechanisms to control the collaborative work. We identified at least seven coordination mechanisms used in support of the outsourcing partnership: the goals identification, the SLAs specification, the KPIs definition, the schedules and guiding principles establishment, the service framework agreement, the expectations definition and communication, and the payments right specification. All these mechanisms were directed to achieve concerted actions and common goals between the two organizations.

The findings suggest that the coordination and the definition of roles and responsibilities, i.e., workflow structure in our model, were the most important topics for the work between Outsourcer and Insourcer. It implies a level of agreement on mutual expectations, thus, it reduces ambiguity in the collaboration for a better operation. As this is a single case study, clearly we cannot generalize results. More data need to be collected over other inter-enterprise collaborations to replicate or counter these case study results. Only then, convincing evidence can be established and results can be considered robust and worthy to generalize. 


\section{Influences of the validation's results}

\subsection{The new VITAL MM's units}

The recommendations obtained from the focus group session and the findings of the case study were used to make decisions on what to change in our MM so that its adequacy and suitability are improved. Our decisions concern both the choice of names for our criteria and their definitions. Fig. 5 shows the relationships among the 'new' units of the VITAL MM. These new units are IS architecture, partnering structure, process architecture, and coordination. The analysis of the results of both the focus group session and the case study supported us to take the following immediate actions:

- we renamed the unit enterprise architecture to IS architecture to better reflect its new scope. This scope is apparent in the new definition, which we present below.

- we renamed the unit workflow structure to partnering structure ${ }^{4}$ to emphasize its contents of how crossorganizational work gets done and who is involved including the definition of roles and responsibilities, and organizational structures.

- we renamed the unit IT/business processes to process architecture to involve both the collaborative IT and business processes without discriminating between these two types.

- we merged the unit IT governance with the unit partnering structure, which meant that the definition of the unit partnering structure incorporates all aspects of what was earlier termed IT governance.

Fig. 5 indicates that in terms of definitions, the units partnering structure and IS architecture are the two which need to be defined from scratch. One of our original units (IT/business processes) was renamed with some minor changes to its definition. The unit coordination remained intact. We defined the new unit partnering structure as the cross-organizational work division, organizational structure, and roles and responsibilities that indicate where and how the work gets done and who is involved. Second, based on [23], we defined the unit IS architecture as the fundamental organization of the information management function of the collaboration enterprises embodied in the information systems that realize this function, their relationships to each other and to the environment, and the principles guiding its design and evolution. Understanding of both partnering

\footnotetext{
${ }^{4}$ This term is taken from the work of Galbraith [14]. It refers to the definition of (i) roles of companies who want to work together as a network, and (ii) work ownership.
}

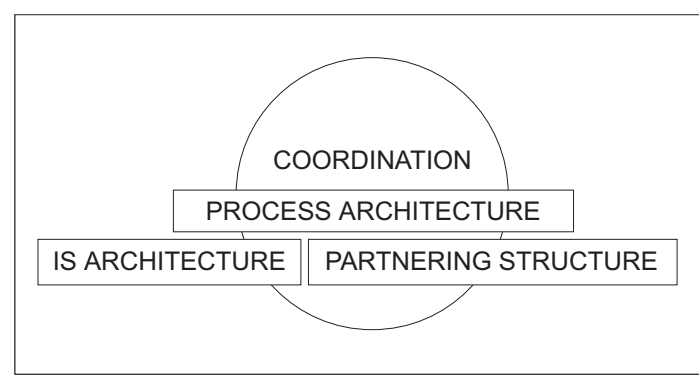

\section{Figure 5. Relationships among the 'new' units of the model.}

structure and IS architecture is needed to efficiently support the process architecture of the inter-enterprise collaboration. Enterprises involved in inter-enterprise businessIT alignment can (re)design the partnering structure and IS architecture separately, however, they need to understand both in order to create and maintain a solid basis for the processes required to achieve shared goals and to exchange information in the inter-enterprise collaboration. Coordination, then, comes next to manage the dependencies among the collaborative activities.

\subsection{New argument to justify the model}

The final concern that three professionals had in the focus group session, i.e., "why not to consider the interenterprise collaborations as single enterprises and then apply Luftman's model for assessing business-IT alignment?", in our opinion stems from a difference in the way of thinking professionals adopt.

There are two kinds of professionals' thinking: bottomup and top-down thinking [5, 12]. The bottom-up thinkers explain phenomena by first looking at higher level selforganized patterns of low level entities. So, they solve a particular problem by first trying out the available theories, methods and tools deeming appropriate. The topdown professionals act the other way around: they would start observing phenomena and, from a particular behavior, they would develop theories, methods and tools to solve the problem. In our research, we apply the top-down way of thinking, as we conjecture that inter-enterprise alignment and the single-enterprise are fundamentally different. The lack of a central decision maker necessitates inter-enterprise collaborations be approached from the perspective of economic theories [28] that simply do not apply in the single enterprise case. Adopting a bottom-up approach, then, poses the danger of overlooking this important issue. 


\subsection{Assessing the validation approach}

Looking back at our experience, we see that the coupling of a focus group session with a case study seems to be a more appropriate approach to validate a maturity model than using either of them in isolation. As identified by Beecham et al. [3] in the development of a Requirements Capability Maturity Model, the three main development stages of a maturity model are: (i) structuring the model, (ii) populating the model with key processes and (iii) validating the model.

Our results show that the multi-method validation approach that we used can help developers of maturity models in assessing their models in the initial development stage. Its main advantage lies in the fact that it offers the best of both merged validation instruments: the focus group helps to gather recommendations from professionals and derive both new insights of the model and changes from design or hypothesis; and the case study helps to obtain useful findings from real-life settings that can also be reflected in the design of the maturity model. A second advantage is due to saving efforts in the case study, i.e., with the insights gained in the focus group session, the case study can easily be conducted with more clarification of the maturity model itself.

\section{Conclusion}

In this paper, we investigated the validity of a maturity model for inter-enterprise business-IT alignment, called the VITAL MM. We used a focus group session and a case study for this purpose. This validation is not the evaluation of the entire model itself. Instead, its sole purpose was to validate the units, i.e., the business-IT alignment criteria, included in the maturity model. We merged focus group and case study methods to form a validation approach that we found helpful in assessing maturity models in their initial stage of development. In our validation process, we experienced the advantages of the complementary use of the two methods. Coupling findings from the focus group session and from a case study provided a much richer context for identifying appropriate refinement actions on our model. Of course, using a multi-method approach could not be enough to overcome external validity concerns that both a case study and a focus group are exposed to. For example, in our case study, the assumptions we make for our maturity model are not representative for all inter-enterprise business-IT alignment projects that exist. We also acknowledge that our case study project from one economic sector may not be representative even for all the business-IT alignment projects that may exist in this economic sector.

The results gained through the two validation instruments used in this study let us increase our knowledge of the completeness and the suitability of the units we included in the VITAL MM. Results of the focus group session suggested that some units were not properly termed or defined. The name of these units caused some confusion among the professionals. Therefore, we decided to rename or merge some of the previously proposed units. We conducted the focus group session prior to the case study so that initial qualitative data were used to have new insights into the units before their identification in the case study. The objective of the case study was, then, to identify in documentation those pieces of information relevant to each of the units, so that we could see what concerns were important for the particular inter-enterprise collaboration studied. The case study findings suggest that coordination and partnering structure are the most important topics to consider when dealing with inter-enterprise settings.

Future work includes replicating our validation process, that is conducting more focus groups and case studies in different inter-enterprise settings to replicate or counter these results and to generalize findings. This will also reveal both advantages and limitations of our validation approach. As we are validating the completeness and suitability of the business-IT alignment criteria included in the VITAL MM, both the focus groups and the case studies will be conducted with professionals and organizations from different economic sectors. That is to make sure we cover distinct settings to extend and/or adjust our criteria and get different insights. Moreover, we will also need to identify what is a good and a not so good practice for each of the units of the VITAL MM to determine their maturity levels. For example, for the unit IS architecture, we need to identify what is the best way to structure the relationships among IT systems so that these create value for the entire collaboration and business-IT alignment is achieved. With this in mind, we plan to investigate what the attributes and the key process areas are that will relate to specific aspects of the IS architecture best practices we found. This validation study therefore served to design a second proposal of the units of the model that need to be evaluated with further validation work.

\section{Acknowledgments}

We are grateful to the professionals who participate in the focus group session for their valuable comments on improving our model, and to the two companies of the interenterprise collaboration studied (who, for reasons of confidentiality must remain anonymous) for their support to this first step in validating the business-IT alignment criteria of the VITAL MM. We also want to thank María Laura Ponisio and Xiaomeng Su who read this paper in draft form providing comments that assisted us in making this paper as sound as possible. 


\section{References}

[1] R. Allen. Workflow: An introduction. In L. Fischer, editor, The Workflow Handbook 2001, pages 15-38. Lighthouse Point, Fla.: Future Strategies, Book Division, 2001.

[2] A. April, J. H. Hayes, A. Abran, and R. Dumke. Software maintenance maturity model $\left(\mathrm{SM}^{m m}\right)$ : the software maintenance process model. Journal of Software Maintenance and Evolution: Research and Practice, 17:197-224, 2005.

[3] S. Beecham, T. Hall, C. Britton, M. Cottee, and A. Rainer. Using an expert panel to validate a requirements process improvement model. Journal of Systems and Software, 76(3):251-275, 2005.

[4] S. Bleistein, A. Aurum, K. Cox, and P. Ray. Strategyoriented alignment in requirements engineering: Linking business strategy to requirements of e-business systems using the soare approach. Journal of Research and Practice in Information Technology, 36(4):259-276, 2004.

[5] L. Boyd. Explanation versus intervention in complex systems, 2003. White Paper.

[6] Y. Chan. Why haven't we mastered alignment? the importance of the informal organization structure. MIS Quarterly Executive, 1(21):76-112, 2002.

[7] M. B. Chrissis, M. Konrad, and S. Shrum. CMMI: Guidelines for process integration and product improvement. Addison-Wesley, 2003.

[8] R. Clotet, X. Franch, P. Grnbacher, L. López, J. Marco, M. Quintus, and N. Seyff. Requirements modelling for multi-stakeholder distributed systems: Challenges and techniques. In C. Rolland, O. Pastor, and J.-L. Cavarero, editors, Proceedings of the 1st International Conference on Research Challenges on Information Science (RCIS'07), pages 413-424, Ouarzazate, Morocco, 2007.

[9] D. R. Cooper and P. S. Schindler. Business Research Methods. Boston, [Mass., etc.]: McGraw-Hill, 8th edition, 2003.

[10] D. Damian. Stakeholders in global requirements engineering: Lessons learned from practice. IEEE Software, 24(2):21-27, 2007.

[11] A. M. Davis and A. M. Hickey. viewpoints: Requirements researchers: Do we practice what we preach? Requir. Eng., 7(2):107-111, 2002.

[12] D. C. Dennett. Cognitive science as reverse engineering: Several meanings of "top-down" and "bottom-up". In D. Prawitz, B. Skyrms, and D. Westerstahl, editors, Proceedings of the 9th International Congress of Logic, Methodology and Philosophy of Science, Uppsala, Sweden, 1991.

[13] Federal Architecture Working Group. Architecture alignment and assessment guide, 2000.

[14] J. R. Galbraith. Designing organizations: an executive briefing on strategy, structure, and process. Jossey-Bass, 1995.

[15] J. Gordijn. Value-based Requirements Engineering: Exploring Innovative e-Commerce Ideas. $\mathrm{PhD}$ thesis, Vrije Universiteit Amsterdam, Amsterdam, The Netherlands, 2002.

[16] J. Hartman. Using focus groups to conduct business communication research. The Journal of Business Communication, 41:402-410, 2004.

[17] A. R. Hevner, S. T. March, J. Park, and S. Ram. Design science in information systems research. MIS Quarterly, 28(1), 2004.
[18] Information Systems Audit and Control Association (ISACA). Cobit: 4th edition, 2006.

[19] H. Jonkers, M. Lankhorst, R. van Buuren, S. Hoppenbrouwers, M. Bonsangue, and L. van der Torre. Concepts for modelling enterprise architectures. International Journal of Cooperative Information Systems (IJCIS ), 13(3):257-287, 2004.

[20] B. Kitchenham, S. Pfleeger, L. Pickard, P. Jones, D. Hoaglin, K. Emam, and J. Rosenberg. Preliminary guidelines for empirical research in software engineering. IEEE Transactions on Software Engineering, 28:721-734, 2002.

[21] B. Kitchenham, L. Pickard, and S. L. Pfleeger. Case studies for method and tool evaluation. IEEE Software, 12(4):5262, 1995.

[22] J. Luftman. Assessing IT-business alignment. Information Systems Management, 20:9-15, 2003.

[23] M. W. Maier, D. Emery, and R. Hilliard. Software architecture: Introducing IEEE standard 1471. Computer, 34(4):107-109, 2001.

[24] F. Niessink. Perspectives on improving software maintenance. $\mathrm{PhD}$ thesis, Vrije Universiteit Amsterdam, Amsterdam, The Netherlands, 2000.

[25] S. L. Pfleeger. Software engineering: Theory and practice. Prentice Hall, second edition, 2001.

[26] H. Prem and G. E. Mathew. How IT enables business transformation? CuttingEdge, Infosys, Jul 2006.

[27] R. Santana Tapia. A value-based maturity model for IT alignment in networked businesses. In CAISE '06: Proceedings of Workshops and Doctoral Consortium of the 18th International Conference on Advanced Information Systems Engineering, pages 1201-1208, Luxembourg, Grand Duchy of Luxembourg, June 2006. Presses Universitaires de Namur.

[28] R. Santana Tapia, M. Daneva and P. van Eck. Developing an inter-enterprise alignment maturity model: research challenges and solutions. Technical Report TR-CTIT-07-29, Centre for Telematics and Information Technology, University of Twente, Enschede, May 2007.

[29] N. Ramasubbu, M. Krishnan, and P. Kompalli. A process maturity framework for managing distributed development. IEEE Software, 22(3):80-86, 2005.

[30] S. Renner. Net-centric information management. In $E D O C$ '06: Proceedings of the 10th IEEE International Enterprise Distributed Object Computing Conference (EDOC'06), pages 265-273, Washington, DC, USA, 2006. IEEE Computer Society.

[31] V. Salaka and V. Prabhu. Project management for enterprise integration. In EDOC '06: Proceedings of the 10th IEEE International Enterprise Distributed Object Computing Conference (EDOC'O6), pages 284-296, Washington, DC, USA, 2006. IEEE Computer Society.

[32] Y. Simmons. Using focus groups as an applied research tool. Howe's Now, 6(2), Apr 2000.

[33] M. W. A. Steen, M. M. Lankhorst, and R. G. van de Wetering. Modelling networked enterprises. In EDOC'02: Proceedings of the Sixth International Enterprose Distributed Object Computing (EDOC'02), pages 109-119, Washington, DC, USA, 2002. IEEE Computer Society. 
[34] A. T. Tao, J. Yang, and H. Jia. Business collaboration development: A case study in capital market. In EDOC '06: Proceedings of the 10th IEEE International Enterprise Distributed Object Computing Conference (EDOC'06), pages 449-452, Washington, DC, USA, 2006. IEEE Computer Society.

[35] J. D. Thompson. Organization in Action. McGraw-Hill, 1967.

[36] P. van Eck, H. Blanken, and R. Wieringa. Project GRAAL: towards operational architecture alignment. International Journal of Cooperative Information Systems, 13(3):235255, Sep 2004.

[37] R. Wieringa. Requirements researchers: are we really doing research?. Requir. Eng., 10(4):304-306, 2005.

[38] R. Wieringa, J. Gordijn, and P. van Eck. Value-based business-it alignment in networked constellations of enterprises. In REBNITA '05: Proceedings of the 1st International Workshop on Requirements Engineering for Business Need and IT Alignment, Paris, France, 2005.

[39] R. K. Yin. Case study research: Design and methods. Applied Social Research Methods Series; vol. 5. Sage Publications, third edition, 2003.

[40] J. Zachman. A framework for information systems architecture. IBM Systems Journal, 26(3):276-292, 1987. 